NASA Technical Memorandum 103640

AIAA-91-0692

\title{
Measurements and Predictions of a Liquid Spray From an Air-Assist Nozzle
}

Daniel L. Bulzan and Yeshayahou Levy

Lewis Research Center

Cleveland, Ohio

and

Suresh K. Aggarwal and Susheel Chitre

University of Illinois at Chicago

Chicago, Illinois

Prepared for the 29th Aerospace Sciences Meeting sponsored by the American Society of Aeronautics and Astronautics Reno, Nevada, January 7-10, 1991

\section{N/NS}




\author{
Daniel L. Bulzan* and Yeshayahou Levy*" \\ NASA Lewis Research Center \\ Cleveland, Ohio $\mathbf{4 4 1 3 5}$ \\ and \\ Suresh X. Aggarwal ${ }^{\dagger}$ and Susheel Chitre \\ The University of Illinois at Chicago \\ Chicago, lllinois 60680
}

\begin{abstract}
Droplet size and velocity and gas velocity were measured in a water spray using a twocomponent Phase/Doppler Particle Analyzer. A complete set of measurements was obtained at axial locations from 5 to $50 \mathrm{~cm}$ downstream of the nozlocations from 5 to 50 cal a simple axi symmetric air-assist nozzle. The sprays produced, using the air-assist nozzle. The sprays produced, using the ters were less than $20 \mu \mathrm{m}$ at all locations. Measurements were obtained for droplets ranging from 1 to $50 \mathrm{\mu m}$. The gas phase was seeded with micron to $50 \mathrm{\mu m}$. The gas droplets, and droplets having diameters of $1.4 \mu \mathrm{m}$ and less were used to represent gas-phase $1.4 \mu \mathrm{m}$ and less were used to represent gas-phase tions from a multi-phase computer model. Initial tions from a multi-phase were taken from measureconts a cm downstream. Predictions for both the gas phase and the droplets showed relatively good agreement with the measurements.

\section{Nomenclature}

a

$\mathrm{C}_{\mathrm{i}}$

$\mathrm{C}_{\mathrm{d}}$ drag coefficient

d nozzle diameter

$d_{p} \quad$ droplet diameter

k turbulence kinetic energy

$\mathrm{L}_{\mathrm{e}}$ dissipation length scale of eddy

mp droplet mass

n number of droplet groups

$n_{i}$ number of droplets per unit time in group $i$

Re Reynolds number

I radial distance

\footnotetext{
*Aerospace Engineer, member AlAA.

- "NRC Research Associate, on leave from Technion, Israel Inst itute of Technology, Haifa,

$f_{\text {Associate professor, Dept. of Mechanical }}$

Engineering, member AIAA. Engineering
} Israel member AIAA.
\end{abstract}

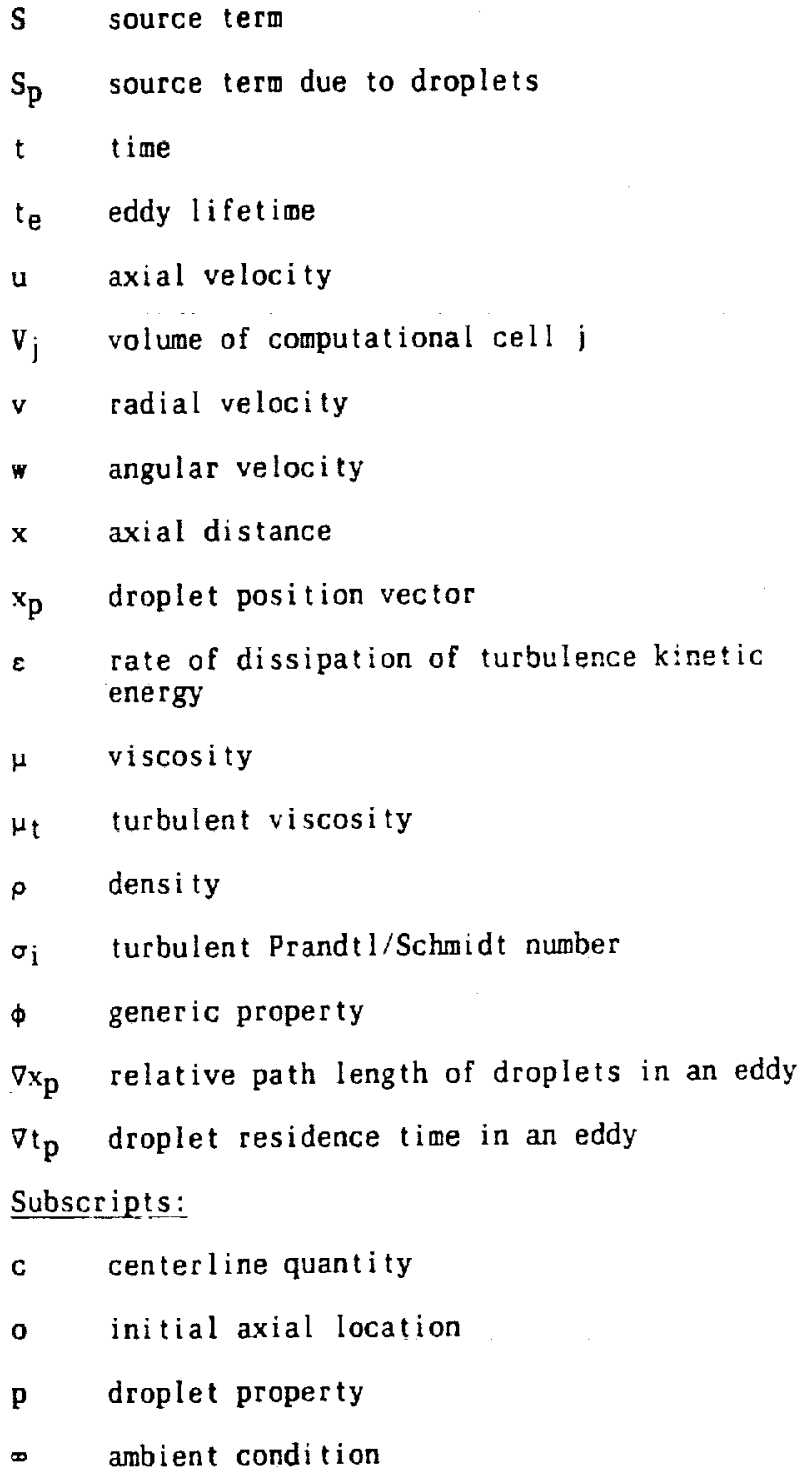

( )' fluctuating quantity

( $)$ time averaged value

$\leftrightarrow \quad$ vector quantity

$(\sim)$ Favre averaged value 


\section{Int roduction}

Spray nozzles have many important applications and have been the subject of considerable research. The objective of the present investigation was to obtain measurements of droplet size, velocity, and volume flux and gas velocity in the flowfield of a simple atomizer and compare their values with predictions from a multiphase computer model.

A similar investigation was performed by Dodge and Schwalb ${ }^{1}$ using a pressure swirl atomizer. Most of the initial conditions for their model were estimated, however, predictions were shown not to be very sensitive to initial conditions. For that study, air velocities were very low in comparison to droplet velocities. A single-component Phase/ Doppler instrument was used for the measurements. The liquid utilized was an aircraft fuel system calibration fluid (MIL-C-7024 typeII). The computer model used for comparison with the data was the FLUENT code. In an earlier study, reported by Solomon, et al., ${ }^{2}$ an air-atomizing nozzle operating with nonevaporating vacuum pump oil was investigated. Gas-phase velocities were measured with a standard LDA system. Droplet size and velocity were measured using a double-flash shadow photographic method. Droplet fluxes were measured using an isokinetic sampling probe. Experimental measurements were compared with predictions from three twophase flow models.

0 ther experimental spray studies using a Phase/Doppler instrument are reported in Refs. 3 and 4. Measurements are presented for both droplet and gas-phase velocities. Presser and Semerjian also report a spray study using an ensemble light scattering technique. No gas-phase measurements are presented. A recent study is reported in Ref. 6 describing Phase/Doppler measurements of a water spray in a swirling flowfield. Both gas phase and droplet measurements are reported.

\section{Experimental Methods}

The sprays were directed vertically downward within a large enclosure ( 1.8 by 1.8 by $2.4 \mathrm{~m} \mathrm{high}$ ) . The air-assist nozzle could be traversed vertically within the enclosure while the enclosure and nozzle could be traversed together in two horizontal directions. This arrangement allowed rigid mounting of all optical instrumentation used during the study. The flow downstream impinged upon a screen located $1 \mathrm{~m}$ below the measurement plane to remove larger droplets and allow smaller droplets to be used as seeding for the ambient air.

The air-assist nozzle is shown in Fig. 1. The inside diameter of the liquid tube was $0.39 \mathrm{~mm}$ and the orifice for the nozzle was $3.18 \mathrm{~mm}$. This nozzle was selected because it is relatively simple and does not utilize a swirler for atomization. Water was used for the liquid spray and was supplied using a tank pressurized with nitrogen to provide a steady flowrate without pressure oscillations. Liquid flowrates were measured using a mass flowmeter. Air flowrates were measured using a $1.27 \mathrm{~mm}$ diameter critical-flow orifice. The air flowrate was controlled by regulating upstream pressure of the air. The atomizing air was seeded with micron sized droplets supplied from a Norgren lubricator. The fluid used for the seeding was Rosco fog fluid, typically used for smoke generators. The average measured drop size in the jet using only the seeder was $1.5 \mathrm{\mu m}$. For the results reported in the present paper, the air and water flowrates were 2.25 and $0.49 \mathrm{~g} / \mathrm{s}$, respectively.

All measurements were performed using a twocomponent Phase/Doppler Particle Analyzer. Previous studies describing this instrument have been reported in Refs. 7 and 8 . The configuration chosen for the present experiment utilized a 2-W argonion laser, frequency shifting for both channels using rotating diffraction gratings, a $500 \mathrm{~mm}$ focal length focusing lens, and a $450 \mathrm{~mm}$ focal length receiving lens. Light was collected $30^{\circ}$ of $f$-axis in the forward direction and focused on to a slit. For measurements at 5 and $10 \mathrm{~cm}$ downstream of the nozzle, a $50 \mu \mathrm{m}$ wide slit was used. The remainder of the measurements used a $100 \mu \mathrm{m}$ wide slit. Generally, 64000 total samples were taken at each measurement location. The number of validated samples varied from about 90 to 20 percent of the total, depending on the region of the flowfield where measurements were being obtained. Measurements in locations near the center of the spray close to the injector exit where number densities were extremely high and velocities were also high showed the largest number of signal rejections. Regions downstream of the nozzle where the spray was not as dense and velocities lower had much higher validation rates. Droplet diameters ranging from 1 to $50 \mu \mathrm{m}$ were measured. Velocity measurements are reported for droplet diameters of $4,11,18$, and $25 \mu \mathrm{m}$. Gas-phase velocities are obtained from measurements of droplets with diameters of $1.4 \mu \mathrm{m}$ or less.. A traverse across the entire spray was performed at each axial measurement station.

\section{Theoretical Me thods}

The analysis is limited to steady, axisymmetric, dilute, nonevaporating, droplet-laden, nonswirling, turbulent jets in an infinite stagnant medium. The boundary-layer approximations are adopted in conjunction with a $k-\varepsilon$ turbulence model for closure. The assumptions for the continuous phase are identical to those reported in Ref. 9. Favre-averaged equations (equivalent to timeaveraged in this case) govern the variation of mean axial and radial velocities, turbulent kinetic energy, and rate of dissipation of turbulent kinetic energy. With these assumptions, the governing equations for the gas phase can be expressed in the following general form:

$$
\begin{aligned}
\frac{\partial}{\partial x}(\hat{\rho} \tilde{u} \phi)+\frac{1}{r} \frac{\partial}{\partial r}(r \tilde{\rho} \tilde{v} \phi)=\frac{1}{r} \frac{\partial}{\partial r}\left[r\left(\mu+\frac{\mu_{t}}{\sigma_{\phi}}\right) \frac{\partial \phi}{\partial r}\right] \\
+S_{\phi}+S_{p, \phi}
\end{aligned}
$$

The parameters $\phi$, and $S_{\phi}$ appearing in Eq. (1), as well as empirical constants used, are listed in Table I.

The turbulent viscosity was calculated as:

$$
\mu_{t}-C_{\mu} \bar{\rho} \frac{k^{2}}{\varepsilon}
$$

Boundary conditions for Eq. (1) are: 


$$
\mathbf{r}=0, \frac{\partial \phi}{\partial r}=0 ; \quad r+\infty, \quad \phi=\phi_{\infty}
$$

Initial conditions were obtained from the measurements obtained at $\overline{5} \mathrm{~cm}$ downstream of the nozzle. $\varepsilon_{0}$ was calculated $f$ rom the definition of a turbulent length scale and is given as follows:

$$
\varepsilon_{0}=C_{\mu} \frac{k^{3 / 2}}{t}
$$

where $L$ was taken as $B$ percent of the flow width at that axial location. Initial values of $w$ were assumed to be equal to measured values of $v^{\prime}$.

The computation of the liquid phase is based on the solution of Lagrangian equations of motion for a statistically significant number of representative droplet groups. Major assumptions for the droplet trajectory calculations are: quasisteady gas phase, no droplet-droplet interaction, and use of a solid-sphere drag law. With these assumptions, the position and velocity of each drop let group can be found by integrating:

$$
\begin{gathered}
\frac{d x_{p i}}{d t}=u_{p i} \\
\frac{d u_{p i}}{d t}=\left(\frac{3 \rho C_{D}}{4 d_{p} \rho_{p}}\right)\left(u_{i}-u_{p i}\right)\left|\vec{u}-\vec{u}_{p}\right|+a_{i}
\end{gathered}
$$

where $i=1,2,3$ for each one of the Cartesian coordinates and the velocities shown in these equations are instantaneous velocities. The drag coefficient was calculated as follows:

$$
\begin{array}{rr}
C_{D}=\frac{24}{\operatorname{Re}\left(1+\frac{\operatorname{Re}^{2 / 3}}{6}\right),} & \operatorname{Re}<1000 \\
C_{D}=0.44, & \operatorname{Re} \geq 1000
\end{array}
$$

The effects of turbulent gas phase fluctuations on droplet trajectory are incorporated using a stochastic separated-flow (SSF) model. The interaction between turbulence and droplets is considered by using a random-walk technique. Each group of droplets interacts with a succession of turbulent eddies. The interaction time is taken to be the minimum of either the eddy lifetime or the transit time for the droplet to cross the eddy. Properties of each eddy are found by making a random selection from the pdf of velocity. Instantaneous eddy veloc$i$ ty is calculated assuming velocity fluctuations are isotropic with a Gaussian pdf having a most probable value found from the local mean velocity and a variance of $2 k / 3$. Details of the procedure can be found in Refs. 2 and 10 . The interaction between the droplets and the gas phase yields source terms in the governing equations for conservation of momentum. They are found by computing the net change in momentum as each droplet group i passes through a computational cell $j$ and is given as follows:

$s_{p, u_{j}}=v_{j}{ }^{-1} \sum_{i=1}^{n} n_{i} m_{p, i}\left(u_{p, i}-u_{p, i} i_{\text {out }}\right)_{j}$ where, $n_{i}$ is the number of particles per unit time in each group. The calculations for the continuous phase were performed using a modified version of Genmix. 11 The computational grid utilized has 33 cross-stream $\mathrm{grid}$ nodes and streamise step size was limited to 2 percent of the current flow width or an entrainment increase of 2 percent - whichever was smaller. For the droplet calculations, 19200 droplet groups were tracked through the flowfield.

\section{Results and Discussion}

\section{Axial Profiles}

The variation of centerline values with axial distance are illustrated in Fig. 2(a) to (e). Both measurements and predictions are presented for axial velocities of both gas and I iquid phases. As illustrated in Fig. 2(a) to (d), axial velocities at $5 \mathrm{~cm}$ downstream of the nozzle are fairly high, ranging from $80 \mathrm{~m} / \mathrm{s}$ for the gas phase to $93 \mathrm{~m} / \mathrm{s}$ for the $25 \mu \mathrm{m}$ drops. As measured, the droplets had higher velocities than the gas at this axial location. Axial velocities decay very quickly from 5 to $20 \mathrm{~cm}$ downstrean of the nozzle for both the gas phase and the three droplet sizes shown. Results for the $4 \mu \mathrm{m}$ droplets were almost identical to the gas phase and are not shown. Even though the droplet size is relatively small, larger droplets possess higher axial velocities at distances less than $20 \mathrm{~cm}$ from the nozzle. At distances greater than about $20 \mathrm{~cm}$, velocity differences between droplet sizes are considerably reduced. As illustrated in Fig. 2, predictions from the model for axial centerline velocities are in reasonably good agreement with the measurements. At downstream distances of 5 and $10 \mathrm{~cm}$, the centerline measurements presented for the $25 \mu \mathrm{m}$ droplets are based on less than 50 samples, consequently they should be used with caution. Sample sizes for the $18 \mu \mathrm{m}$ and smaller drops were adequate at all downstream locations. At larger downstream distances, centerline measurements for the $25 \mu \mathrm{m}$ droplets are based on at least 2000 samples. Figure $2(e)$ presents the axial centerline profile of turbulence kinetic energy for the gas phase, normalized by the local centerline axial velocity. For the experimental values. since only $u^{\prime}$ and $v^{\prime}$ were measured, it was assumed that $w$ was equal to $v^{\prime}$. The increase of normalized turbulent kinetic energy with axial distance is initially overpredicted, however, predictions show better agreement with the measurements at increasing axial distances from the injector. No attempt was made to account for the modification of the gas-phase turbulence by the droplets.

\section{Radial Profiles}

Radial profiles of spray properties at $10 \mathrm{~cm}$ downstream are illustrated in Fig. $3(a)$ to (f). Results are presented for the $18 \mathrm{~mm}$ dianeter droplets and the continuous phase. Radial profiles for the other droplet sizes were similar except for the fluctuating quantities, which will be discussed later. Gas phase radial profiles of mean axial velocity, $k$, and $u^{\prime} v^{\prime}$ are shown in $\mathrm{Fig}, 3(\mathrm{a})$ to (c). Centerline axial velocity has decayed to about $43 \mathrm{~m} / \mathrm{s}$ and is underpredicted by about 20 percent. Centerline $k$ and peak values of $u^{\prime} v$ are 
somewhat overpredicted at this axial location, however the jet width is fairly well predicted. Predicted and measured radial profiles of mean axial velocity, and fluctuating axial and radial velocities of $18 \mu$ miameter droplets are illustrated in Fig. $3(d)$ to (f). Again, the peak axial velocity is underpredicted, but only by about 10 percent. Fluctuating axial velocities are substantially greater than fluctuating radial velocities at this axial location. Predicted values of fluctuating axial velocity show lower values than the measurements, while fluctuating radial values agree reasonably well with the measurements. This is due to the assumption of isotropic fluctuating velocities in the model, while measured axial fluctuating velocities were larger than radial fluctuating velocities. The predicted values show some scatter in the profiles. Predictions for the 18 um droplets were obtained using 4800 droplet groups. Increasing the number of droplet groups would result in smoother radial profiles. Larger droplets showed larger fluctuating axial velocities than smaller droplets near the centerline at this axial location. however, this may be due to the fact that fewer larger droplets are found at the center of the spray and the sample size may not be large enough. An examination of the complete data set showed that larger droplets consistently showed lower fluctuating radial velocities across the entire width of the spray than smaller droplets. This was also shown by the predictions. Fluctuating radial velocities are important for turbulent dispersion of the droplets since they are larger than the mean radial velocities in the present spray.

Radial profiles at $20 \mathrm{~cm}$ downstream of the nozzle are illustrated in Fig. $4(a)$ to (f). Results are again presented for the gas phase and $18 \mu \mathrm{m}$ diameter droplets. Figure 4 (a) to (c) presents gas phase measurements and predictions of mean axial velocity, $k$, and $\overline{u^{\prime} v}$. The centerline gas velocity has decayed to about $22 \mathrm{~m} / \mathrm{s}$ from the value of $80 \mathrm{~m} / \mathrm{s}$ measured at $5 \mathrm{~cm}$ downstream of the nozzle. The predictions agree reasonably well with the measurements; the centerline is underpredicted by about 6 percent. Radial profiles of $k$ and also $\overline{u^{\prime} v^{\prime}}$ show fairly good agreement with the measurements at this axial location, al though $k$ is underpredicted. Radial profiles of the $18 \mu \mathrm{m}$ droplets are presented in Fig. 4(d) to (f). Even at $20 \mathrm{~cm}$ downstream of the nozzle, mean axial velocities of the droplets are slightly, but consistently, larger than the gas phase. Peak centerline velocity is underpredicted by about 4 percent, however values elsewhere show very good agreement with the measurements. Fluctuating axial and radial velocities of the $18 \mu \mathrm{m}$ droplets again show effects of anisotropic velocity fluctuations, but are reduced here compared to values observed at $10 \mathrm{~cm}$ downstream of the nozzle. Predicted radial fluctuating velocities agree with the measurements, while axial fluctuating velocities are still lower than the measurements.

Profiles at $30 \mathrm{~cm}$ downstream are shown in Fig. 5(a) to (f) for both the gas phase and the 18 $\mu \mathrm{m}$ droplets. Cas phase axial centerline velocity has decayed to about $14 \mathrm{~m} / \mathrm{s}$. Predictions of mean axial velocity show excellent agreement with the measurements at this location. Gas phase $k$ and u'v are also shown, where some scatter is evident in the $u^{\prime} v^{\prime}$ data. The reason for the scatter is probably due to a measurement inaccuracy at this location. Droplet profiles at this axial location are also presented for the $18 \mu \mathrm{m}$ droplets. Predic- tions for mean axial velocity and fluctuating radial velocity show quite good agreement with the measurements. Predictions of fluctuating axial velocity again are lower than the measurements, however, the differences are decreasing as axial distance increases and axial velocity decreases.

Radial profiles at the largest axial downstream measurement location of $50 \mathrm{~cm}$ are presented in Fig. 6(a) to (f). As illustrated in Fig. 6(a) to (c), predictions of gas phase radial properties show fairly good agreement with the measurements at this location, although $k$ is still underpredicted, especially near the centerline. The data show a slight shift to the positive $T$ direction of about $2 \mathrm{~mm}$ at this axial location. This is probably due to a slight misallignment between the nozzle axis and the gravity vector which is more evident at larger axial distances. Radial profiles for the 18 um diameter drops are presented in Fig. 6(d) to (f). Centerline mean axial droplet velocity has decayed to slightly less than $10 \mathrm{~m} / \mathrm{s}$ at this axial location. Fluctuating radial velocity is still smaller than fluctuating axial velocity, however, the flow becomes more isotropic. Predicted values show the same distribution trends as the measurements, but values are on the order of 20 percent lower than the measurements.

Radial profiles for the $\downarrow, 11$, and $25 \mu \mathrm{m}$ droplets are not presented. Results for the $4 \mu \mathrm{m}$ droplets are almost identical to the gas phase. The results for the $11 \mu \mathrm{m}$ droplets are somewhere between the gas phase and the $18 \mu \mathrm{m}$ droplets. The $25 \mathrm{um}$ droplets show larger differences between the drops and the gas phase, but there are relatively few of these in the spray, especially near the center of the jet near the nozzle.

\section{Conclusions}

A complete set of measurements and predictions of both the gas phase and droplets have been presented for a water spray spraying downward into stagnanant surroundings. The flow geometry was kept relatively simple so that an axisymetric, parabolic, computer model could be used. Major conclusions from the study are listed as follows:

1. Axial centerline velocity decay with distance downstream of the nozzle was reasonably well predicted for both the gas phase and the droplets. For the $18 \mu \mathrm{m}$ diameter droplets, droplet mean axial velocities decayed from $86 \mathrm{~m} / \mathrm{s}$ at $5 \mathrm{~cm}$ downstream of the nozzle to $9 \mathrm{~m} / \mathrm{s}$ at $50 \mathrm{~cm}$ downstream. The gas phase decayed from $80 \mathrm{~m} / \mathrm{s}$ at $5 \mathrm{~cm}$ to $9 \mathrm{~m} / \mathrm{s}$ at $50 \mathrm{~cm}$ downstream of the nozzle.

2. Predicted radial profiles of mean properties for both the gas phase and the droplets showed relatively good agreement with the measurements at all axial stations. Better agreement was obtained as axial distance increased.

3. For the $18 \mu \mathrm{m}$ droplets, fluctuating axial velocities were underpredicted while fluctuating radial velocities generally showed good agreement with the measurements. This was probably caused by the isotropic assumption of the SSF model.

4. The spray produced by the nozzle was very axisymmetric making it useful for comparison with model predictions. 


\section{References}

1. Dodge, L.G., and Schwalb, J.A., "Fuel Spray Evolution: Comparison of Experiment and CFD Simulation of Nonevaporating Spray," Lournal of propulsion and Power. Vol. 111, 1989, pp. 15-23.

2. Solomon, A.S.P., Shuen, J.-S., Zhang, Q.-F., and Faeth, G.M., "Structure of Nonevaporating Sprays: I. Near-Injector Conditions and Mean Properties," AIAA Journal, Vol. 23, No. 10, Oct. 1985, pp. 1548-1558.

3. McDonelI, V.G., and Samuel sen, G.S., "Evolut ion of the Two-Phase Flow in the Near Field of an Air-Blast Atomizer Under Reacting and NonReacting Conditions," Fourth International Symposium on Applications of Laser Anemometry to Fluid Mechanics, Lisbon, Portugal, 1988.

4. McVey J.B., Kennedy J.B., and Russell, S., "Fuel-Injector/Air Swirl Characterization," NASA CR-180864, 1988.

5. Presser, C., and Semer jian, H.G., "Dynamics of Pressure-Jet and Air-Assist Nozzle Sprays: Aerodynamic Effects," AIAA-Paper 88-3139, June 1988.

6. Breña de la Rosa, A., Wang, G, , and Bachalo, W.D., "The Effect of Swirl on the Velocity and Turbulence Fields of a Liquid Spray," ASME Paper 90-CT-32, 1990.
7. Bachalo, W.D., Rudoff, R.C., and Houser, M. J.. "Laser Velocimetry in Turbulent Flow Fields: Particle Response," AIAd-Paper 87-0118, Jan. 1987.

8. Bachalo, W.D., Rudoff, R.C., and 8reña de La Rosa, 1. , "Vass Flux Measurements of a High Number Density Spray System Using the Phase Doppler Particle dnalyzer," AIAA-Paper 88-0236. Jan. 1988.

9. Shuen, J.-S., Solomon, A.S.P., and Faeth, G.M., "Drop-Turbulence interactions in a Diffusion Flame," AlAA Journal, Vol. 24, Jan. 1986. pp. $101-108$.

10. Faeth, G.M., "Evaporation and Combust ion of Sprays," Progress in Energy and Combustion Science, Vol. 9, No. 1-2, 1983, pp. 1-76.

11. Spalding, D. B., GENMIX: A Ceneral Computer Program for Two-Dimensional Parabolic Phenomena. Pergamon Press, 0xford, 1978.

TABLE 1, - SOURCE TERMS IN GOVERNING EQUATIONS

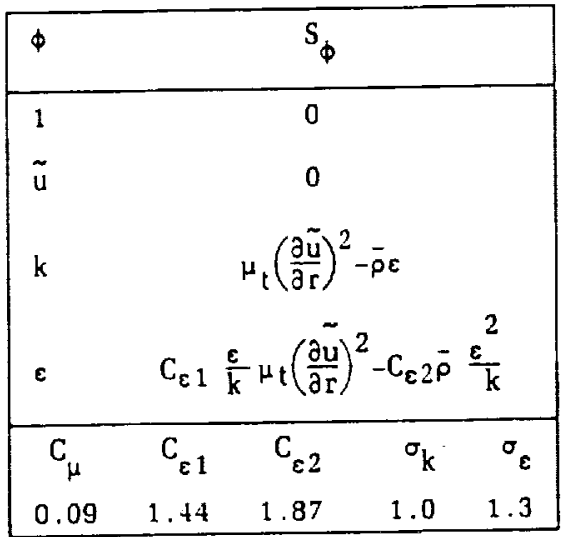

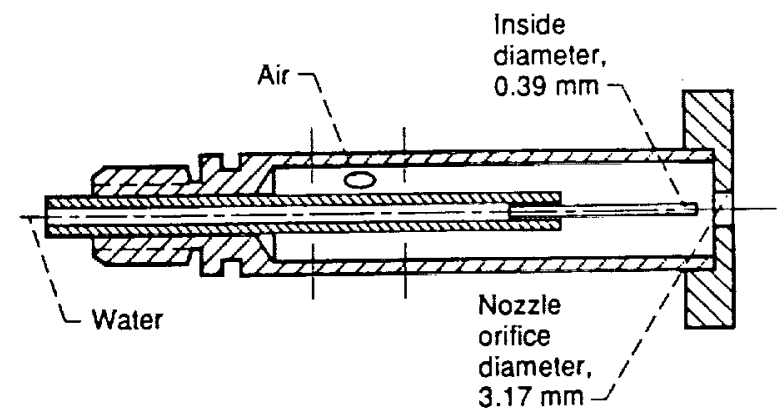

Figure 1.-Diagram of air-assist nozzle. 


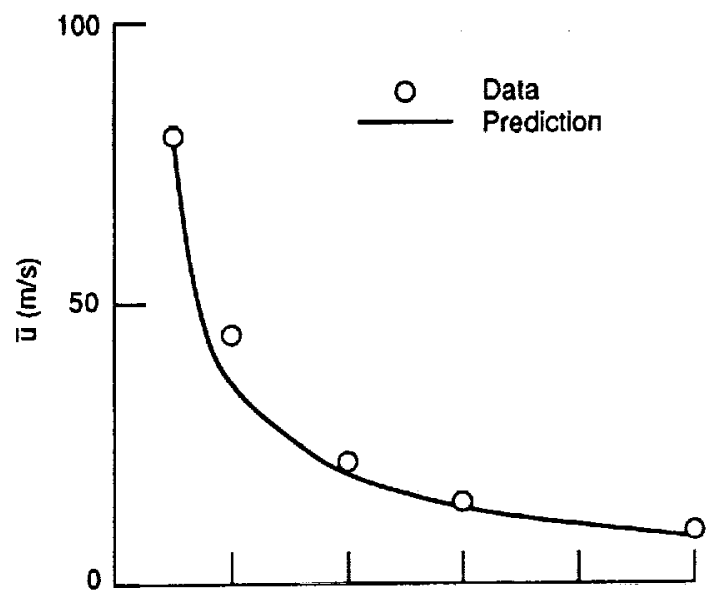

(a) Gas phase.

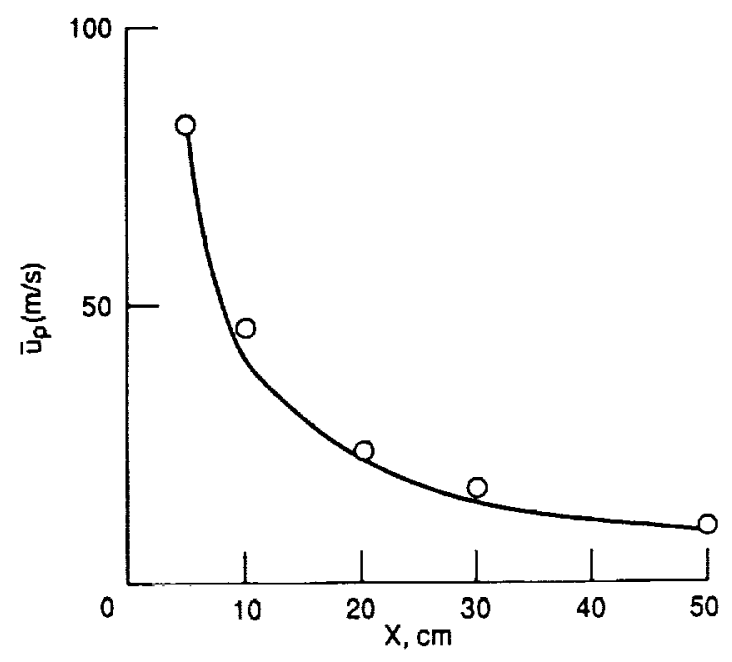

(c) 18 Micron drops.

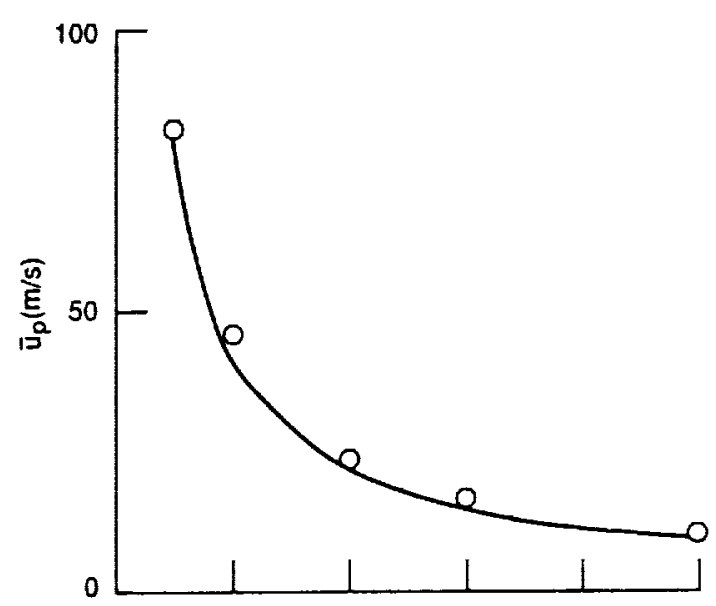

(b) 11 Micron drops.

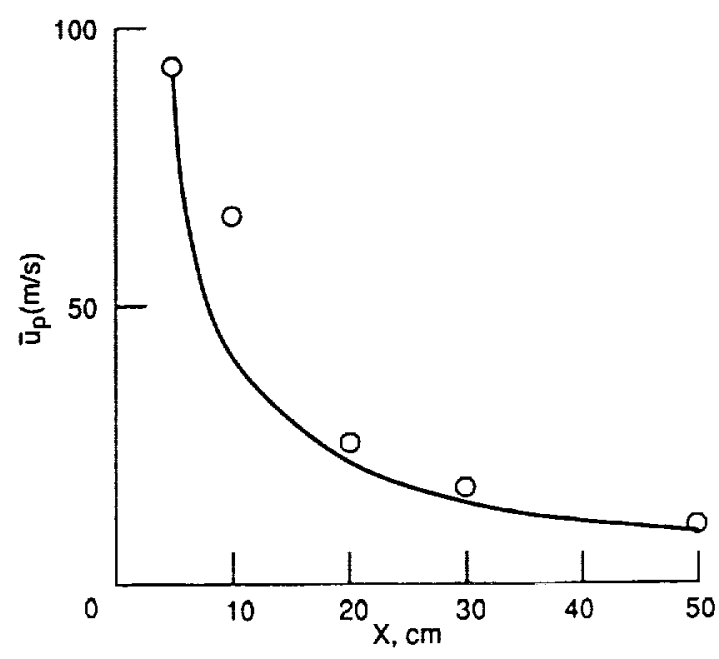

(d) 25 Micron drops.

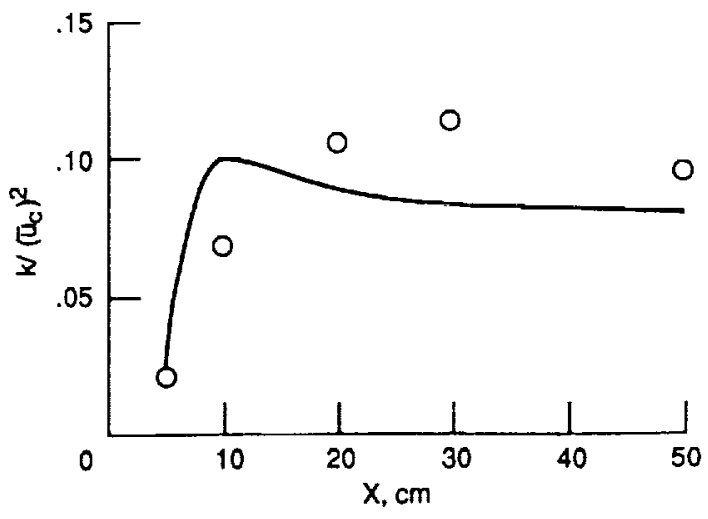

(e) Gas phase furbulence kinetic energy.

Figure 2.-Axial variation of centerline properties. 


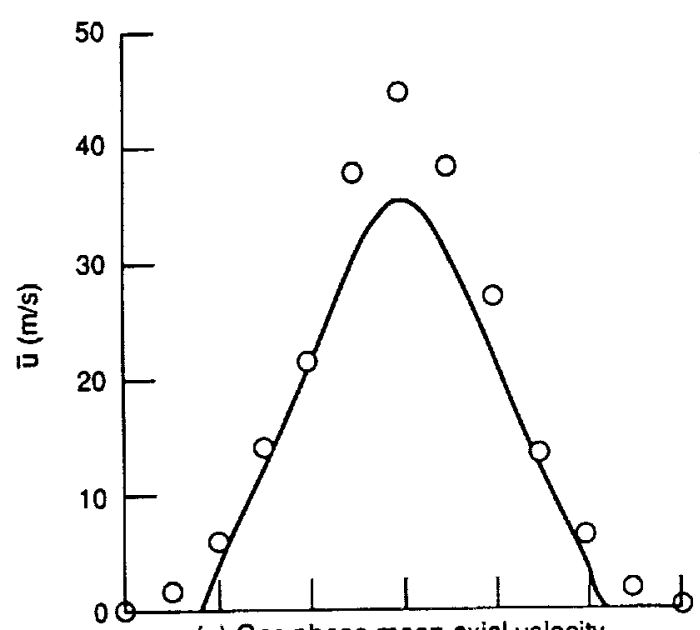

(a) Gas phase mean axial velocity.
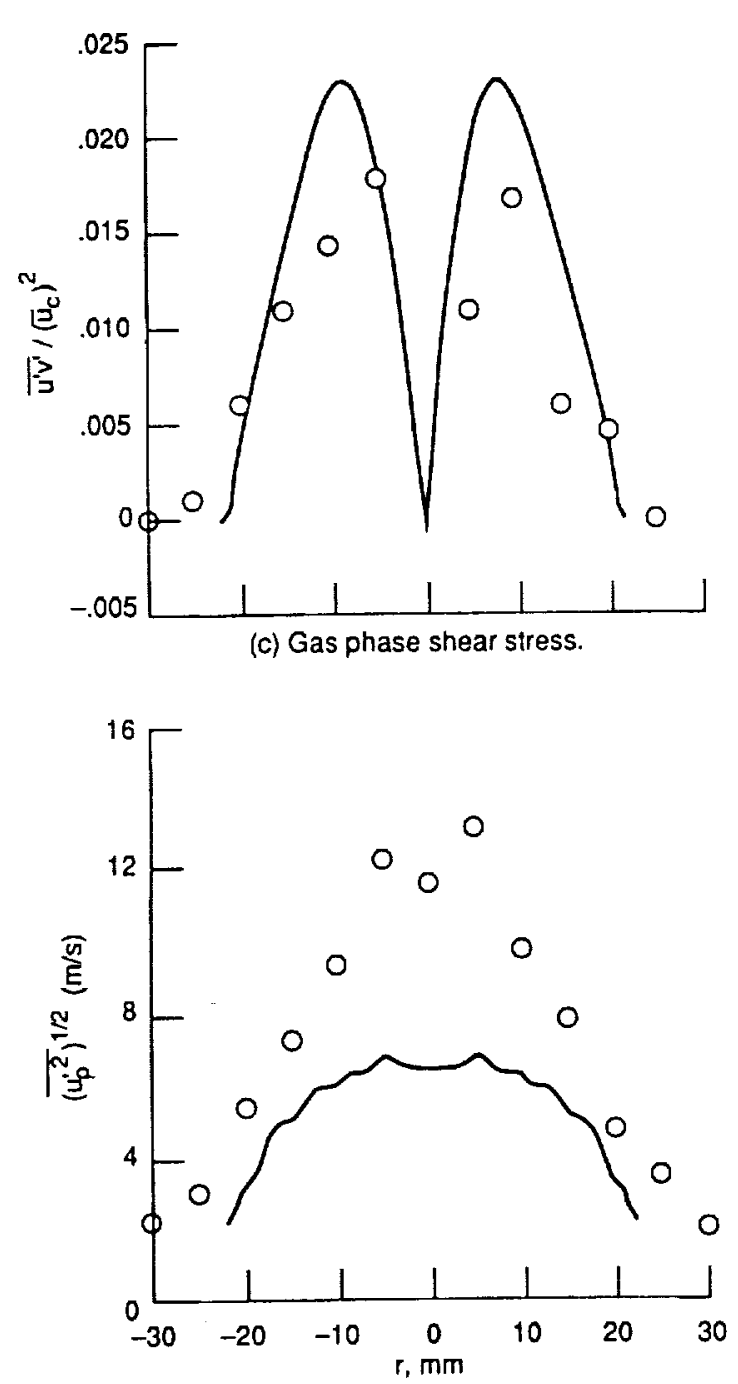

(e) Fluctuating axial velocity for 18 micron drops.
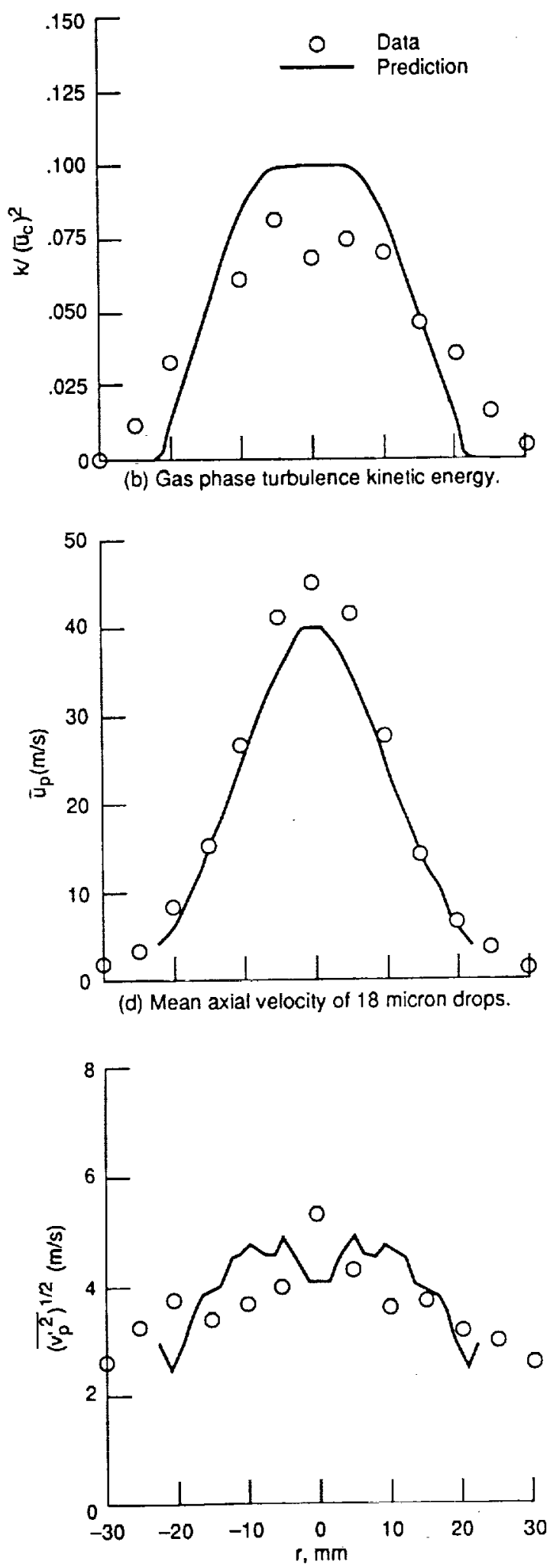

(f) Flucluating radial velocity for 18 micron drops.

Figure 3.-Radial profiles af $10 \mathrm{~cm}$ downstream of the nozzle. 

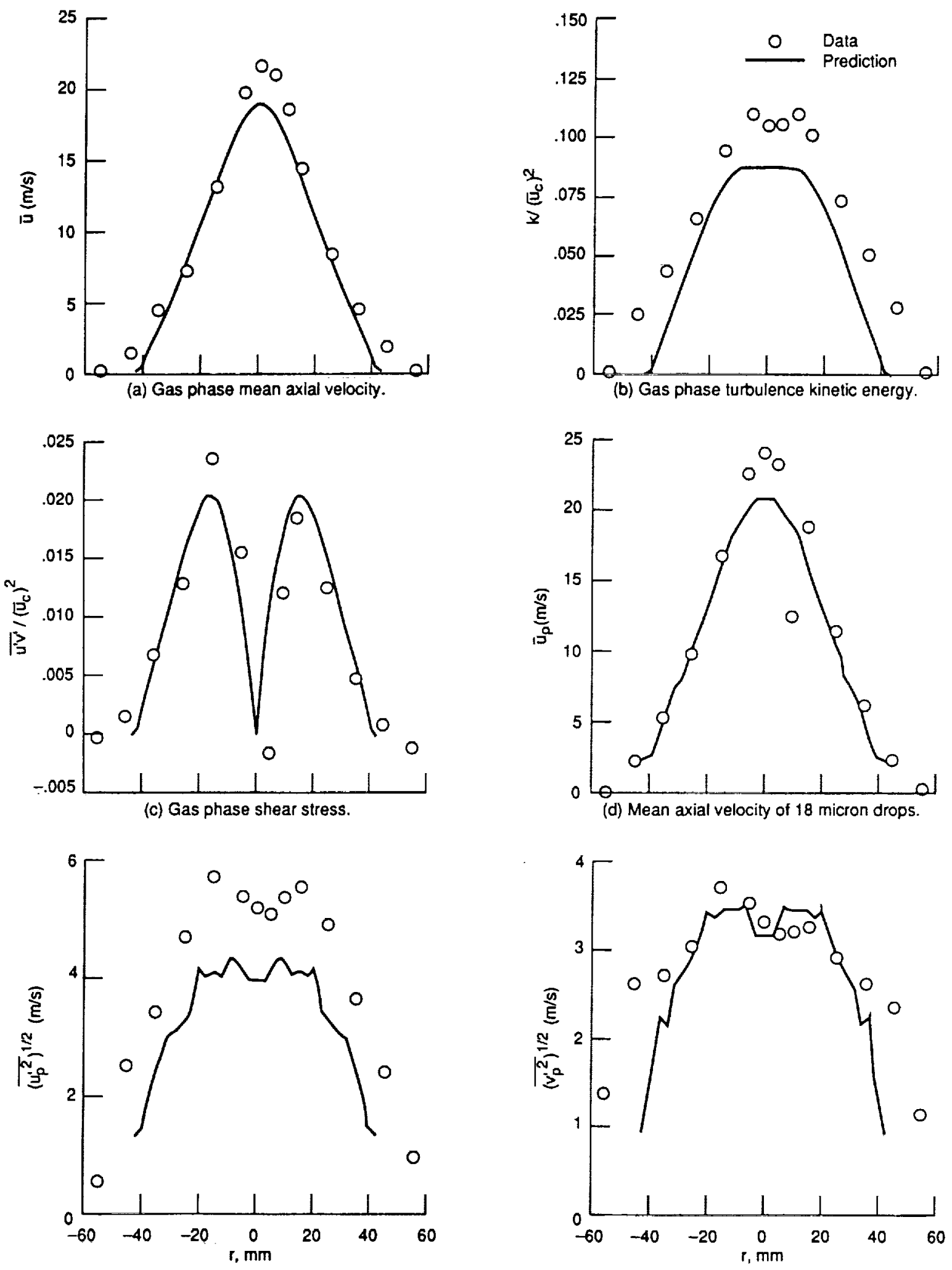

(e) Fluctuating axial velocity for 18 micron drops.

(f) Fluctuating radial velocity for 18 micron drops.

Figure 4.-Radial profiles at $20 \mathrm{~cm}$ downstream of the nozzle. 


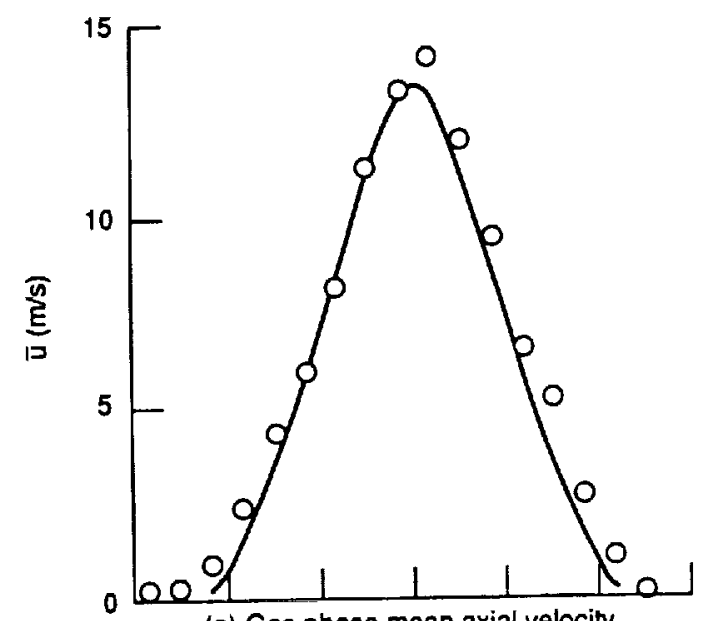

(a) Gas phase mean axial velocity.

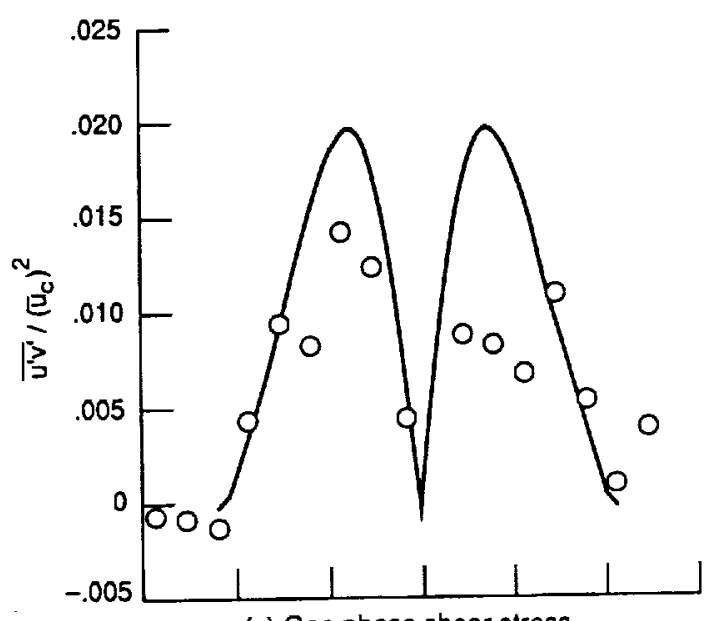

(c) Gas phase shear stress.

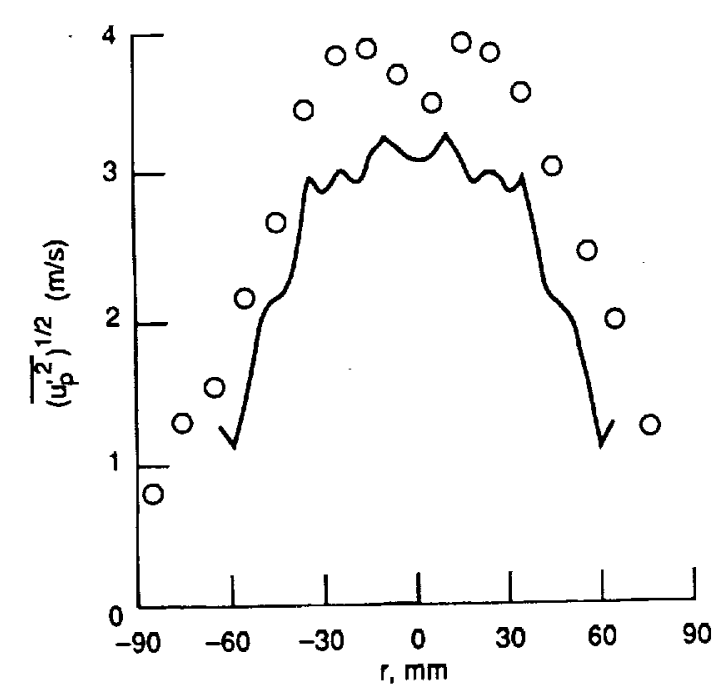

(e) Fluctuating axial velocity for 18 micron drops.
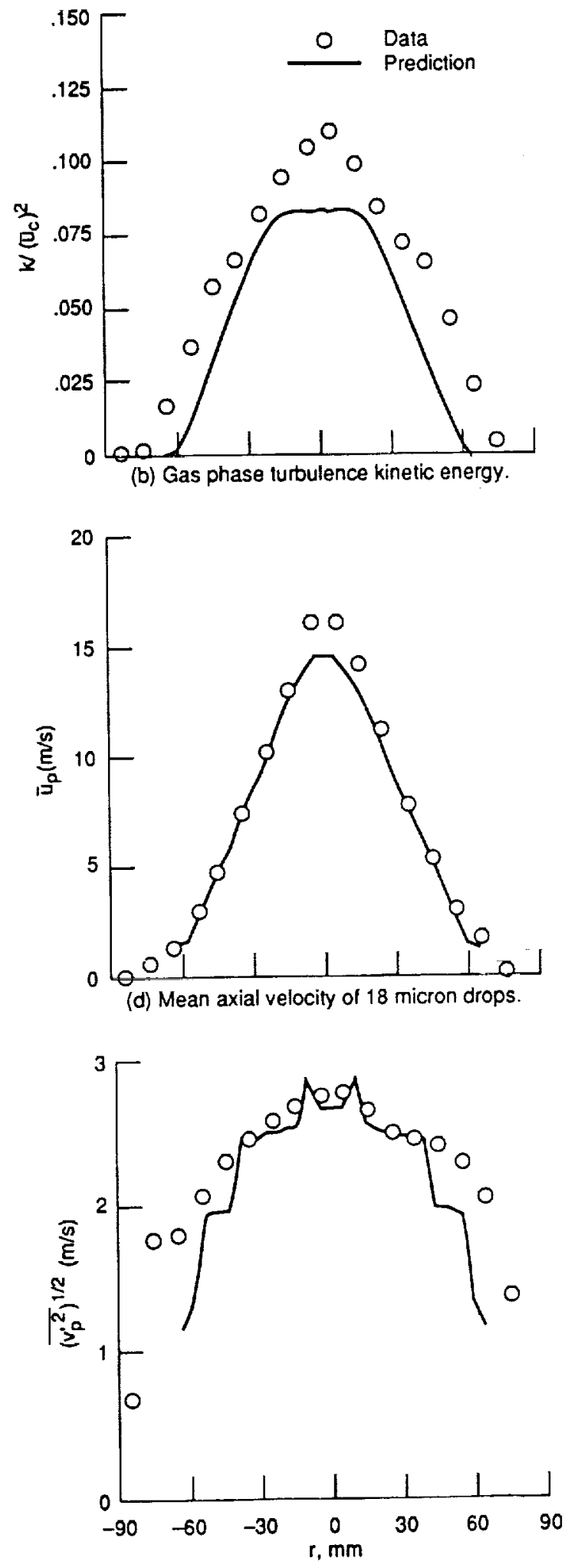

(f) Flucluating radial velocity lor 18 micron drops.

Figure 5.-Radial profiles at $30 \mathrm{~cm}$ downstream of the nozzle. 


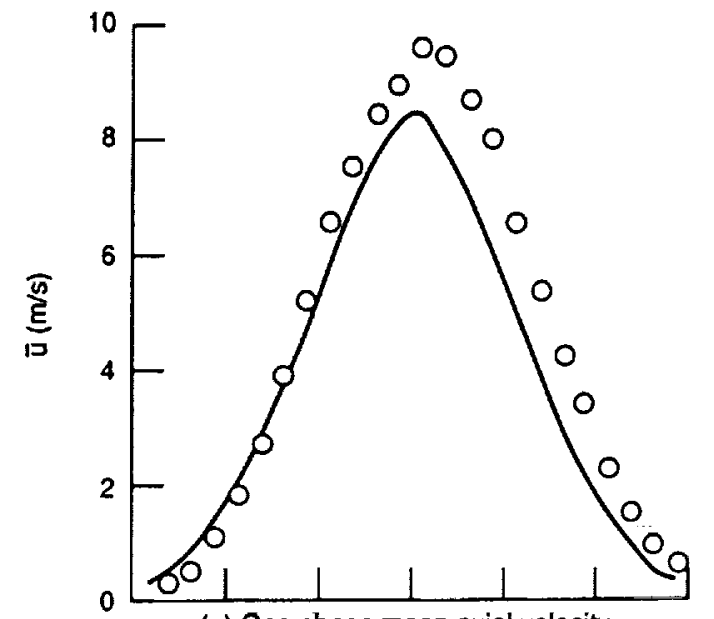

(a) Gas phase mean axial velocity.

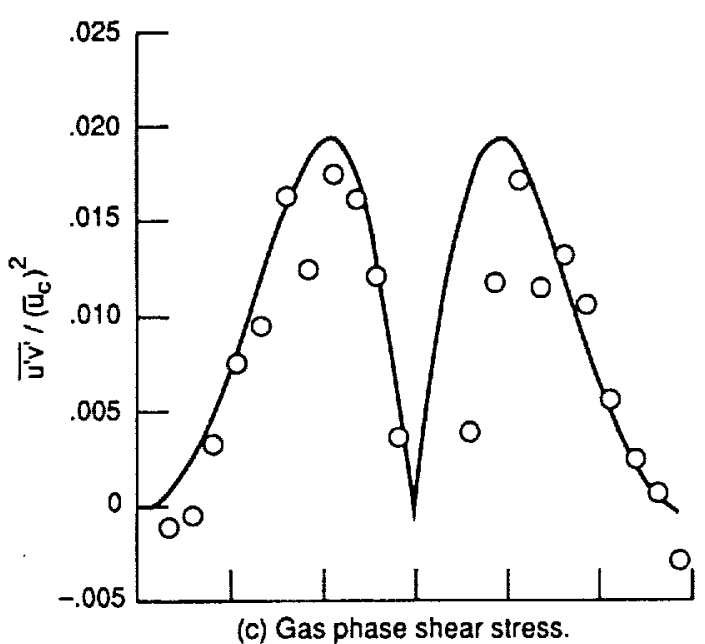

(c) Gas phase shear stress.

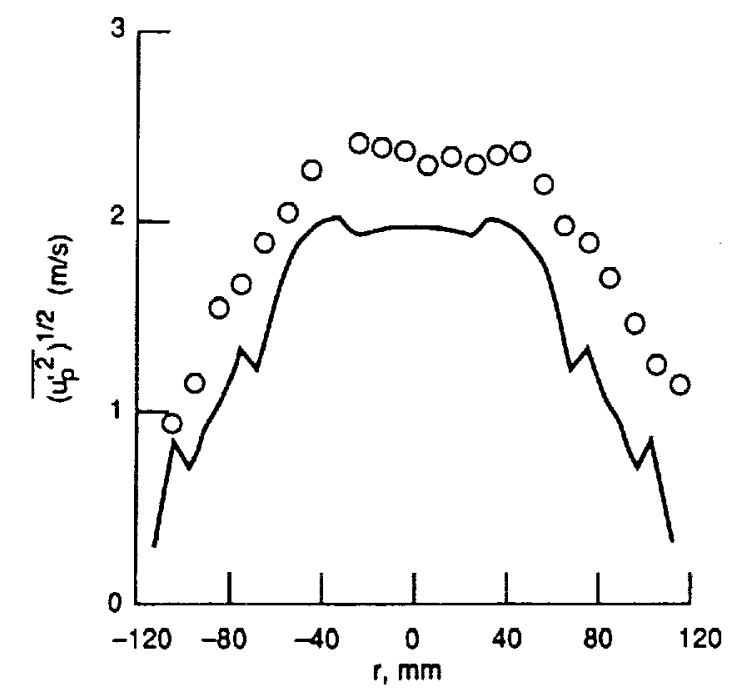

(e) Fluctuating axial velocity for 18 micron drops.

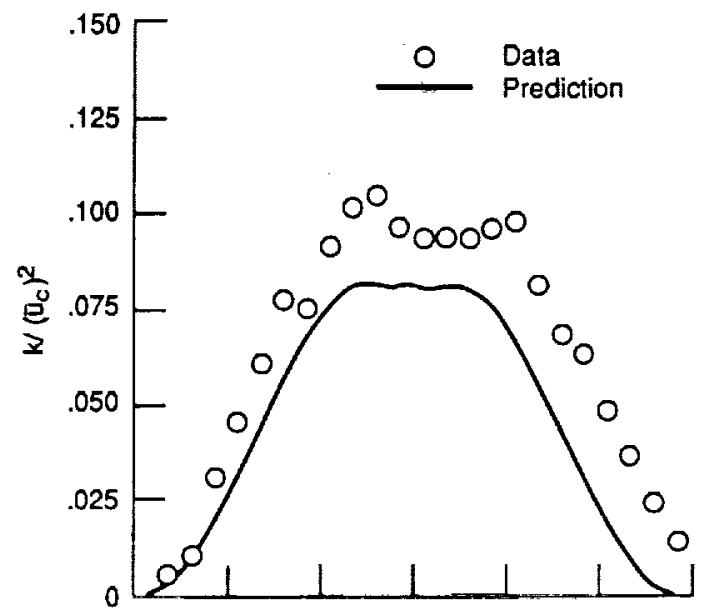

(b) Gas phase turbulence kinetic energy.
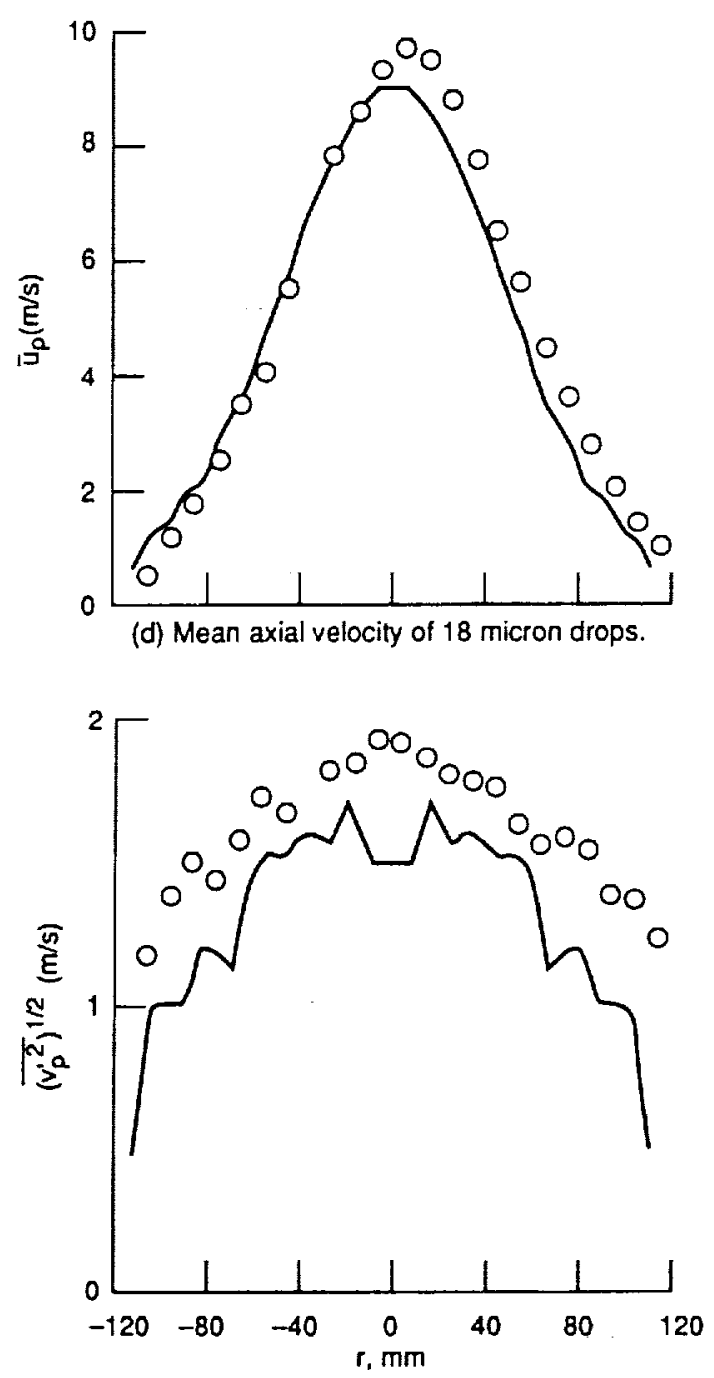

(f) Fluctuating radial velocity for 18 micron drops.

Figure 6.-Radial profiles at $50 \mathrm{~cm}$ downstream of the nozzle. 


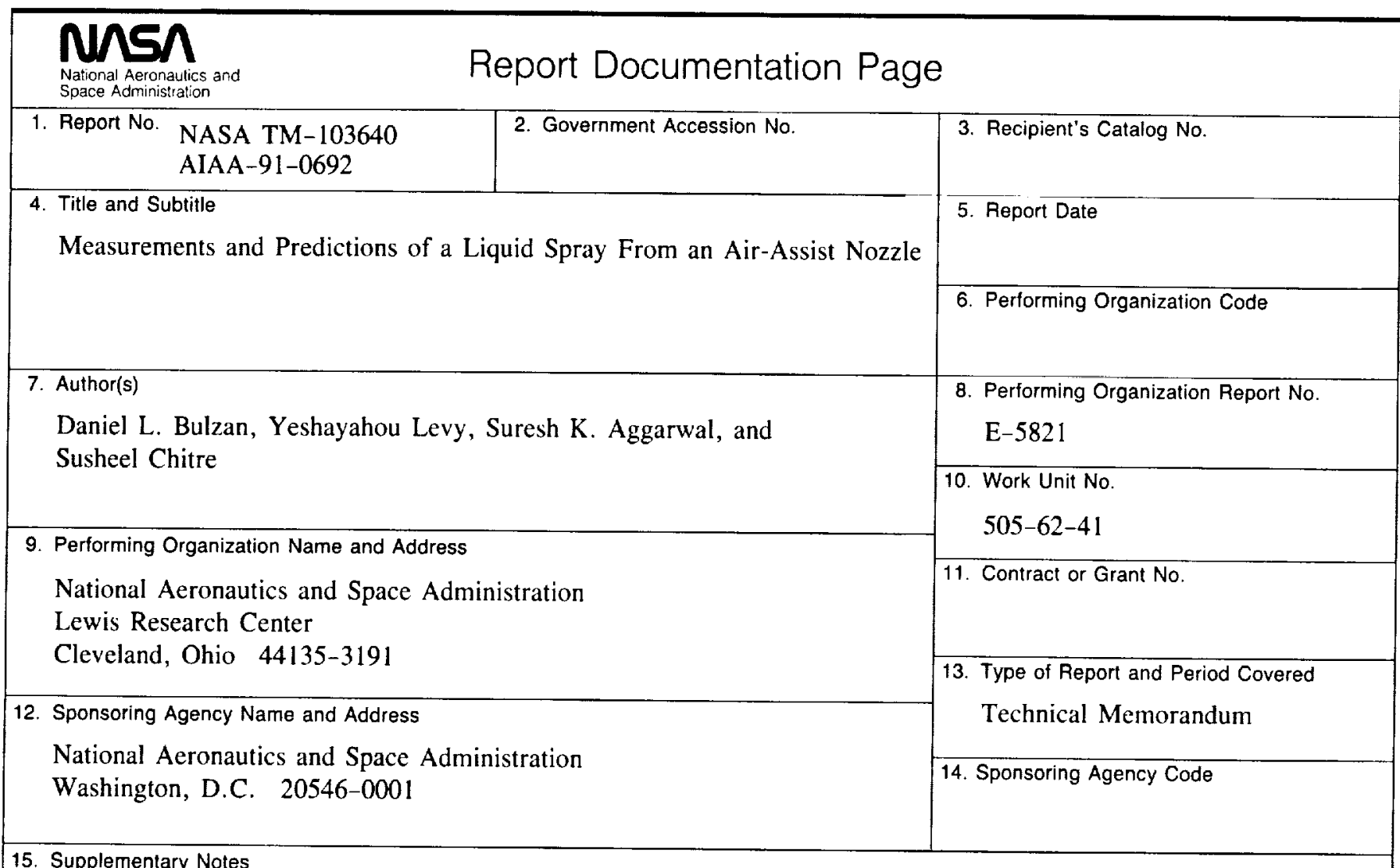

Prepared for the 29th Aerospace Sciences Meeting sponsored by the American Society of Aeronautics and Astronautics, Reno, Nevada, January 7-10, 1991. Daniel L. Bulzan, NASA Lewis Research Center. Yeshayahou Levy, National Research CouncilNASA Research Associate, on leave from Technion, Israel Institute of Technology, Haifa, Israel. Suresh K. Aggarwal and Susheel Chitre, University of Illinois at Chicago, Dept. of Mechanical Engineering, Chicago, Illinois 60680

16. Abstract

Droplet size and velocity and gas velocity were measured in a water spray using a two-component Phase/Doppler Particle Analyzer. A complete set of measurements was obtained at axial locations from 5 to $50 \mathrm{~cm}$ downstream of the nozzle. The nozzle used was a simple axisymmetric air-assist nozzle. The sprays produced, using the atomizer, were extremely fine. Sauter mean diameters were less than 20 microns at all locations. Measurements were obtained for droplets ranging from 1 to 50 microns. The gas phase was seeded with micron sized droplets, and droplets having diameters of 1.4 microns and less were used to represent gas-phase properties. Measurements were compared with predictions from a multi-phase computer model. Initial conditions for the model were taken from measurements at $5 \mathrm{~cm}$ downstream. Predictions for both the gas phase and the droplets showed relatively good agreement with the measurements.

17. Key Words (Suggested by Author(s))

Sprays

Multiphase flow
18. Distribution Statement

Unclassified - Unlimited

Subject Category 07
19. Security Classif. (of this report)

Unclassified
20. Security Classif. (of this page)

Unclassified
21. No. of pages

13
22. Price*

A03


